Fecha de recepción: febrero 2017 Fecha de aceptación: agosto 2017 Versión final: septiembre 2018

\section{La función social del diseño o el diseño al servicio social}

Jorge Gaitto *

Resumen: La función del diseño no debería ser ni cuestionada, ni convertirse en el eje de una discusión, ya que a priori su misión manifiesta es social. Surge desde la sociedad y su producción está orientada y dirigida hacia la sociedad, y configurada de acuerdo a las necesidades temporales del contexto del cual emerge. Sin embargo, abordar el tema de la funcionalidad supone definirla de manera más precisa: ¿es social o humanitaria? El presente trabajo aporta una mirada sobre estas cuestiones, e intenta reflexionar acerca del posible deber ser del diseño frente a los diferentes posicionamientos profesionales.

Palabras clave: diseño social - diseño humanitario - diseño efímero.

[Resúmenes en inglés y portugués en la página 29]

${ }^{*}$ Diseñador Gráfico. Prof. Titular Regular de Tipografía I y II FADU / UBA. Secretario Académico de la Facultad de Diseño y Comunicación de la Universidad de Palermo. Docente de Postgrado (Maestría en Gestión del Diseño UP). Conferencista. Crítico. Tutor de Tesis de Grado y Posgrado.

\title{
Introducción
}

Lejos de toda obviedad, es necesario destacar que el diseño, como actividad, nace en el seno de la sociedad y su producción está orientada y dirigida hacia ella. Por este motivo su función no puede ser sino social. Es en la sociedad donde incide,, tanto en forma positiva como negativa. En este sentido, no se puede ignorarse que son producto del diseño tanto las bombas como los campos de refugiados. (Chaves, 2006, p. 15)

Las especificidades de la función social que el diseño adquiere se definen y varían de acuerdo a las necesidades temporales de la comunidad en la cual se desarrolla. Puede plantearse, el caso particular de proyectos cuyos fines son útiles para mejorar la calidad de vida de las personas o que se desarrollan sólo y únicamente como respuesta y cobertura de las necesidades humanas básicas.

Sin embargo, considerar la disciplina como una actividad con fines propios y autónomos, sería olvidar que se trabaja con propósitos específicos definidos por un cliente, que es quien genera la demanda del servicio. Y que el perfil del diseño en una sociedad, no está condicionado únicamente por los intereses de quienes lo demandan, sino también por 
los de quienes mandan; que -curiosamente y en la gran mayoría de los casos- son coincidentes.

No es el diseño el generador autónomo de su función social sino que es el diseñador como integrante activo de su sociedad. Dicho de otro modo: no es el diseño, son las políticas. Un ejemplo de esto es que una suntuosa torre de viviendas en una exclusiva zona, es tan producto de diseño como un barrio de viviendas populares de bajo coste destinado a las poblaciones de menores recursos.

Si bien, el diseño es un motor importante de las economías emergentes, del total de la masa crítica de los diseñadores de estos países, sólo un $2 \%$ dedica parte de su tiempo profesional a entidades sin fines de lucro (vinculando su producción hacia la función social y humanitaria directamente) y el restante $98 \%$ se encuentran trabajando en sectores de servicios, comerciales e industriales.

Dentro de este último grupo, y en el marco del fenómeno del emprendedorismo y del auto-empleo, han proliferado las denominadas industrias del diseño. Son, por lo general, empresas pequeñas o medianas que ofrecen servicios de diseño o donde el diseño es uno de los elementos centrales: ya sea se trate de publicidad, moda, interiorismo o gráfico, entre otros. Venden, asesoran y resuelven problemas de diseño para distintos clientes con los más diversos propósitos, principalmente, con el de conseguir aumentar la venta de productos en el mercado. De esta forma, el diseño influye directamente y de manera profunda en los procesos de innovación, y pude potenciar la competitividad de un país más allá de sus límites, contribuyendo al desarrollo de productos y a su incorporación a los mercados, y garantizando oportunidades laborales a aquellos que con su trabajo calificado generan un valor añadido. ¿Es esto función social?

En el valor agregado que la disciplina da a los productos, muchas empresas o grupos empresarios ven la oportunidad de aplicar políticas de producción verde o de reciclado, malentendiendo el sentido de la Responsabilidad Social Empresaria-RSE. Es por lo menos perverso, tal como sostiene Wolf, proponer la aparición de nuevos productos -mayoritariamente innecesarios- para intentar minimizar el ciclo de producción, consumo y desecho. Los mercados se saturan, bajo esta lógica, de cantidades de objetos realizados con materiales de descarte; intentando generar una reutilización de aquella basura, convirtiéndola en otro deshecho pero esta vez "diseñado". Cabe destacar que, desde la dimensión ética (no ya del diseño sino la de los diseñadores), será una decisión personal para qué cliente trabajar y qué proyectos realizar.

El desafío del desarrollo responsable y consciente de la profesión lleva a reflexionar sobre el contexto en el que se desenvuelve. En este sentido, tal como sostiene Gustavo Valdés de León, "la práctica del diseño no se realiza en un espacio ideal, aséptico, políticamente neutral e incontaminado, sino en un espacio histórico, en contextos fuertemente condicionados por factores económicos, políticos y culturales que se determinan mutuamente" (Valdés de León, 2010, p. 54). Es decir, los diferentes escenarios condicionan el ejercicio efectivo de la disciplina y terminan influyendo sobre diseñadores, sobre la generación de los productos diseñados, y sobre la sociedad que los contiene y de la cual forman parte. Diseñar significa establecer entre el pensar y el hacer una mutua referencia. Y la estética sin ética se aproxima al fraude. Lo que interesa es el producto en su integridad, no únicamente 
su forma exterior o su función. El criterio del uso incluye también los efectos sociales y ecológicos que genera.

Otl Aicher sostiene:

El diseño se relaciona a la situación cultural de una época, al tiempo, al mundo. El mundo actual se caracteriza por su hallarse en permanente proyecto. La civilización actual es algo que han hecho, y por tanto proyectado los hombres. La calidad de los proyectos es la calidad del mundo. (1994, p. 12)

Es decir, el diseño -como actividad profesional- necesita del pleno compromiso de todos los participantes; por lo tanto no todo cliente es adecuado, ni todo diseñador es garantía de resultados de calidad. En este sentido, El diseño permanente e integrado es una de las áreas principales de intervención para los profesionales conscientes de su rol social.

Pierre Bernard (1991), en su conferencia El núcleo del entendimiento, menciona a dos tipos de diseño, y en particular y en lo que refiere al diseño gráfico habla del panorama típico del diseño gráfico permanente y del diseño gráfico efímero.

Vincula al primero con la arquitectura, el diseño urbano, los periódicos, los libros, los mapas, los planos o los sistemas pictográficos; y tiene como característica el trabajo hacia la expresión visual de un desarrollo estructural general. El objetivo de este tipo de diseño se logra cumplir cuando se integra el mensaje (forma, contenido) a la sociedad, confirmando los valores preestablecidos. Transforma la idea, el juicio y el valor estético en una realidad "natural" tangible. Generalmente, se presenta al diseño gráfico como funcional, pero el rol simbólico que juega pronto se vuelve permanente y asume una nueva funcionalidad: la integración ideológica.

El diseño gráfico efímero, a diferencia del permanente, parece incluir actos diversos y específicos: narraciones y afirmaciones contradictorias, divergentes u opuestas sobre afiches, avisos, vidrieras, panfletos, programas o exhibiciones. Este tipo de diseño, de corta vida, apunta a la transmisión de mensajes aislados, vinculados a situaciones específicas y parece tener una naturaleza operativa opuesta a la del diseño gráfico integrado y permanente. Sus productos son efímeros. Atraen la atención y luego desaparecen.

Si es válida esta clasificación (permanente contrapuesto a efímero, e integrado confrontado a independiente), es posible extraer la conclusión de que el consenso ideológico se relaciona con la estabilidad sociográfica. Es decir, las personas tenderán a pensar de la misma forma si el panorama visual es estructurado e inalterable; y la sensación de libertad se vinculará con la presencia de numerosos y variados desacuerdos con respecto a los intercambios vivos. No obstante, como sostiene Berger, ese despliegue de libertad es mera ilusión, cuando la estrategia general que yace por detrás conduce exclusivamente al consumo.

El diseñador debe definir su posición y tomar conciencia de las distintas realidades sociales existentes, incluso de aquellas que surgen de las diferentes situaciones y culturas (locales, regionales, nacionales o internacionales). Se vuelve necesario unir lo efímero y lo permanente, lo integrado y lo independiente para obtener una concepción articulada, compleja, que no sea elitista, ni populista ni reduccionista. De este modo, el diseñador 
gráfico social se relaciona con la dimensión cultural del mensaje y con su articulación en un proyecto de desarrollo cultural a largo plazo, en lo que lo permanente/integrado (estrategia) y lo efímero/independiente (táctica) no están en contradicción.

Oponiéndose a la estandarizada profusión de la publicidad, es necesario trabajar a partir de situaciones sociales particulares, en función a sus dinámicas específicas y sus posibles dimensiones humanas. En base a estas premisas es que aún pequeñas unidades de comunicación podrán construir trabajos creativos que generarán, desarrollarán y agregarán valor a la cultura visual de una sociedad.

¿Es el diseñador el autor solitario de sus obras? La noción de la autoría compartida surge como esencial desde el punto de vista ético. La necesaria cooperación entre el diseñador y el cliente, llevará a compartir con el cliente su posición estética (no carente de ideología), y llevará al diseñador a aceptar la validez de la postura del cliente. Es en este particular equilibrio entre coautores el que permite que la producción se oriente hacia un acto cultural que, por definición, siempre es riesgoso, e implica un profundo compromiso.

En este contexto, se vuelve necesario escuchar más y mejor a las personas a quienes se dirigen nuestros objetos de diseño, más allá de los objetivos, generando una forma de diseñar comprometida con la solidaridad comunitaria y tratando de satisfacer al destinatario. Claramente, esto constituye una actitud y una posición política frente al campo social, que no desatiende el hecho de que el escenario donde se realiza la práctica del diseño es un espacio fuertemente condicionado por diversos factores.

Los aspectos ético-morales del diseño son requerimientos que deberían estar más allá de toda discusión. Es necesario que impere un sistema de reglas y valores que trascienda lo individual en el trato a otros individuos, aún cuando ese sistema varíe de una sociedad -o nación- a otra. Es decir, una región pobre no puede tener las mismas prioridades que se observan una nación rica e industrializada. "Primero la comida y después la moral", tal como sostenía Bertolt Brecht (1928).

Otra paradoja vinculada a la ética del co-diseño es la generación de condiciones de producción ecológicas en el mundo tecnológico. Por un lado se reconoce como una prioridad. Por otro lado, sin embargo, es muy difícil para los diseñadores la creación de nuevos estándares porque éstos suelen estar totalmente distantes de las prioridades del comitente. El contexto fuerza al diseñador a ser oportunista, dado que su existencia y subsistencia como profesional depende de poder proveer un servicio.

La contribución social del diseño se alcanzará concientemente, cuando se logren cortar los lazos con los fines puramente materiales; evitando la superproducción de objetos, tomando debida nota de las otras cuestiones que suceden en nuestras sociedades, tratando de traer de regreso a la profesión (si es que alguna vez la tuvo) cierta aspiración protagónica y activa de su rol social. Es decir, logrando que la actividad del diseño deje de restringirse a los intereses del usuario-consumidor, y pueda recuperar el concepto humano de utilidad. Esto podrá sucederse cuando el ejercicio profesional se lleve adelante más allá de las instrucciones dadas por cliente (que deberán ejecutarse de la mejor manera posible), sino también -y sobre todo- pensando en la sociedad, intentando ver que el trabajo realizado es parte de un asunto más complejo y global, y tomando conciencia sobre las consecuencias directas e indirectas, a corto, mediano y largo plazo de lo que se está diseñando en ese momento. 


\section{Algunas aproximaciones}

En este escenario, cabe preguntarse cómo es posible llevar adelante una profesión vinculada a los proyectos de diseño, cuando se es subsidiario de un cliente, que pertenece a una sociedad de consumo, y cuyo fin primordial es generar mejores ventas para acumular mayores ganancias. ¿Es utópico el pensar que existen alternativas?

Si así fuera, atravesar el campo de la utopía, aún sabiendo de qué se trata, puede colaborar en la toma de decisiones y en la elaboración de alternativas.

Hace más de tres décadas, Alvin Toffler en su texto La tercera ola, ya planteaba la diferencia de los recursos finitos de la tierra, la propiedad o el capital, en relación a los inagotables como el conocimiento (que se puede transferir). "La clave es comprender el futuro, no por curiosidad sino por supervivencia”, sostenía. (Toffler, 1980)

La salida es la educación, en todos los niveles, claro está; pero aquí la referencia es sobre el diseño y la formación de los diseñadores. Hay que pensar en el valor político y ético de la docencia y la producción del saber.

La decisión es política y es social, y compete a todos los actores de la sociedad a la que pertenecen y en la que conviven.

La economía circular ha llegado para romper el paradigma lineal que imperaba desde la Revolución Industrial, cuando el mundo no tenía problemas de abastecimiento y nadie hablaba de extinción de recursos no renovables, de la emisión de dióxido de carbono, del calentamiento global o de la necesidad de una producción sustentable. La anterior perspectiva entendía tanto que los recursos eran eternos y se podían extraer sin consecuencias; como que los productos podían usarse y tirarse.

El crecimiento humano, el impacto ambiental y el consumo, marchan hacia una colisión, en la que el planeta no tendrá recursos ni siquiera para abastecer la demanda de energía, agua o alimentos.

En la economía lineal, el diseño no interviene sobre los residuos; en cambio, si lo hace en la economía circular, porque ese residuo puede ser insumo de otro proceso o remanufacturado.

El belga Gunter Pauli, autor de la Economía azul (Pauli, 2011) y uno de los máximos exponente de la economía circular, ejemplifica ideas y experiencias para desarrollar negocios sostenibles, cuyas transformaciones, además de generar empleo permiten la reutilización de los productos "diseñados" en otra etapa del proceso.

Así es posible que los pañales biodegradables usados se conviertan en tierra negra para sembrar árboles frutales, teniendo en cuenta que un bebé de un año, con sus pañales genera una tonelada de tierra fértil al año; o construir viviendas a partir de botellas de vidrio, o producir papel sin cortar un árbol y sin utilizar agua.

Un ejemplo argentino de la economía circular es la empresa Xinca, de la provincia de Mendoza, que fabrica zapatillas a partir del reciclado de caucho de neumáticos en desuso y la reutilización de deshechos de telas.

En este sentido, diversos gobiernos han tomado medidas para incentivar la reutilización y el reciclaje. Es el caso del gobierno sueco que propone reducir los impuestos sobre las reparaciones de todo tipo de productos. El objetivo es que los consumidores sientan que no tiene sentido deshacerse de los artículos viejos o rotos para comprar nuevos, si pueden 
repararlos a bajo costo. El gobierno confía en que la reducción de impuestos sobre los aparatos de uso doméstico estimule una nueva industria de servicio de reparación hogareña, generando así los tan necesarios puestos de trabajo para los inmigrantes recientes sin educación formal.

Los incentivos forman parte de un cambio de visión del gobierno, que pasó de limitarse a reducir las emisiones de dióxido carbono en el país, a buscar también la reducción de emisiones que generan los productos fabricados fuera de Suecia. Entre 1990 y hoy, Suecia ha reducido sus emisiones anuales de dióxido de carbono en un $23 \%$. Actualmente, más de la mitad de su energía eléctrica proviene de fuentes renovables. (Orange, 2016)

Por otra parte, en Holanda, en octubre de 2009, Martine Postma creó la Fundación Repair Café; una iniciativa donde voluntarios se reúnen a reparar gratuitamente ropa, muebles, electrodomésticos, bicicletas, juguetes, y a luchar contra la obsolescencia programada. La tendencia se ha expandido y actualmente hay más de 1200 cafés repartidos en más de 30 países de todo el mundo. La organización estima que, sólo en 2015, se repararon 200.000 aparatos en todo el mundo y que con esta acción se evitó también la emisión de unos 200.000 kilos de CO2 al ambiente (repaircafe.org). Los Repair Cafés tiene presencia sobre todo en Europa y comienzan a tener una fuerte presencia en América Latina.

Los altos costos de reparación y la obsolescencia programada son parte del mismo fenómeno. En este sentido, el documental Comprar, tirar, comprar (Dannoritzer, 2011) muestra la problemática de los objetos que han sido diseñados para no durar, movilizando la economía pero también incentivando el consumo innecesario y aumentando la contaminación del ambiente. Los Repair Cafés nacen como una opción válida, que crece, para ir en contra de esta tendencia.

Un ejemplo de cambio en la cosmovisión desde el inicio del proceso de creación es Pica Para Arriba, un estudio argentino de Diseño Gráfico Social que trabaja exclusivamente en proyectos que tiene fines sociales, humanitarios, medioambientales o culturales de organizaciones no lucrativas; promoviendo un diseño responsable (picaparaarriba.org).

Los casos anteriores operan como paliativo pero la verdadera operación de cambio debe producirse en el inicio del proceso, donde clientes y diseñadores deberán tomar debida nota, y conciencia, que la producción de objetos (industriales, gráficos, electrónicos, etc.) destinados al consumo de una sociedad, devengará en otros objetos que necesariamente deberá reciclar, reelaborar, destruir o desechar. Teniendo presente no solo su utilidad sino también su inutilidad posterior.

\section{La creatividad como aporte social}

El diseño social muchas veces genera innovación, aunque esta no siempre produce un impacto significativo en la sociedad. Dos casos concretos de impacto social en Latinoamérica son las iniciativas de diseño social realizadas en el barrio argentino de Zavaleta y el mexicano de Colonia Palmitas. 
El primer ejemplo fue realizado por la agencia Young \& Rubicam-Argentina en 2011, cuando diseñó una estrategia innovadora y de alto impacto para mostrar la situación de la villa 21-24. También conocida como barrio de Zavaleta, es la villa miseria más grande y con más población de la ciudad de Buenos Aires. Para principios de 2016, se calculaba que el asentamiento ya tenía más de 50.000 habitantes. (Infobae, 18 de mayo de 2016)

La iniciativa se realizó en conjunto con la Zavaleteros, una organización no gubernamental que trabaja con niños y adolescentes en situación vulnerable. Combinando una estrategia BTL con el diseño social, pintaron de amarrillo flúor las casas del barrio. La campaña estuvo guiada por el lema "Acá estamos" y tuvo el objetivo de generar conciencia sobre la situación precaria del que barrrio en los vecinos de la ciudad de Buenos Aires. (Adlatina, 9 de mayo de 2011)

En este sentido, es interesante como una operación básica como la lectura (y el consiguiente "resaltado" de parte de un texto para recordar o marcar lo más importante) puede ser trasladada un espacio ciudadano, recalcando la parte más importante de un "texto urbano". El segundo ejemplo fue llevado adelante, en 2014, en la Colonia Palmitas de la ciudad mexicana de Pachuca de Soto, estado de Hidalgo. La iniciativa fue creada por la Secretaría de Gobernación, en el marco del programa Nos Mueve la Paz, en conjunto con el colectivo de muralistas Germen Crew. Por un año se trabajó colectivamente -1800 vecinos en beneficio de 452 familias- en la realización de un macro-mural, con el objetivo de recuperar de los espacios urbanos del cerro. El mural fue pintado sobre más de 200 casas, con 190 colores diferentes, con la técnica de graffiti. Según datos de la Secretaria de Planeación y Evaluación del Ayuntamiento de Pachuca, la indicativa tuvo un impacto positivo, disminuyendo notablemente la violencia y las actividades delictivas (nosmuevelapaz.org).

\section{Conclusiones}

El mundo es maravilloso y la realidad fantástica.

Si se hiciera una revisión, en el estricto sentido del término, o sea de re-mirar, se podría establecer algo así como una visión cotidiana del estado del arte.

Si pudiéramos reducir la población de la tierra a una aldea con sólo cien habitantes, pero con los mismos porcentajes humanos actuales, obtendríamos el siguiente resultado: habría 57 asiáticos, 21 europeos, 14 habitantes de la Américas y 8 africanos. La mitad de la riqueza del mundo estaría en manos de sólo seis personas. Los seis serían de nacionalidad norteamericana. Ochenta vivirían en casas de calidad inferior. Setenta serían iletradas. Cincuenta estarían desnutridas. Una estaría a punto de fallecer y otra apunto de nacer. Sólo una entre las cien personas, tendría educación universitaria y ninguna tendría computadora (Diario El País, Madrid).

Este es el mundo que habitamos, donde el incremento sostenido en la última década ha hecho que el gasto mundial en armamentos haya alcanzado un récord histórico, con un incremento del 34\% más que hace diez años, con la impresionante cifra de 1,12 billones de dólares, el 50\% de esa cifra le corresponde a Estados Unidos. Y significativamente la organización mundial Save the Children, informa que el drama del trabajo infantil, creció seis veces en nuestro país desde 1998, afectando a 1.500 .000 chicos. 
Esto parece ser el laberinto de una crisis y como decía inteligentemente Leopoldo Marechal: "de los laberintos hay que salir por arriba".

La llave de esa salida "por arriba" es la educación, para poder luchar de una manera más igualitaria contra los sistemas, la mejor y más confiable herramienta es la educación.

El economista argentino Tomás Bulat (1964-2015), ha hecho en público algunas reflexiones, que pueden colaborar para esclarecer y reafirmar esta idea, cuando sostuvo que "educar es más difícil que enseñar, porque para enseñar Ud. necesita saber, pero para educar Ud. precisa SER". Y se puede agregar para completar la referencia que "cuando se nace pobre, estudiar, es el mayor acto de rebeldía contra el sistema. El saber rompe las cadenas de la esclavitud".

Esta reflexión, es válida en todos los niveles de la cadena educacional, pero ajustando la mirada, sobre las formaciones profesionales del campo proyectual, supone una necesidad de asumir la responsabilidad social, para poder quebrar esa asimetría.

En diferentes regiones de nuestro vasto continente, se ha perdido, durante los últimos años, la estrechez de la relación diseñador/urgencia social. Nos hemos deleitado, al principio, y agotado, al final, en la discusión diseño - arte. No por impropia, pero si por innecesaria, en tanto desviación de los objetivos sociales más importantes y hasta urgentes. El diseño debe ser considerado una como herramienta social. Sin embargo, la gran mayoría de las universidades, en su declaración en cuanto a visión y misión, propone "centrar las miras en el sujeto que aprende, para potenciar al máximo sus capacidades". Y es absolutamente lógico que así sea, pero del mismo modo deben los profesionales "centrar las miras en el sujeto social, tratando de potenciar al máximo sus capacidades”.

Se trata de una revisión (re - visión) y de recordar (re - cordis, volver a pasar por el corazón -según Eduardo Galeano en el Libro de los Abrazos) cuál es nuestra tarea en ésta sociedad, cuál es nuestro rol social, y trabajar más estrechamente con las personas, tratando de hibridar la concepción del diseño con la necesidad social.

\section{Referencias Bibliográficas}

Adlatina (9 de mayo de 2011). Young \& Rubicam presenta Resaltador, su acción para Zavaleteros. Disponible en: http://www.adlatina.com.ar/publicidad/young-rubicam-presentaresaltador-su-acci\%C3\%B3n-para-zavaleteros

Aicher, O. (2005). El mundo como proyecto. Barcelona: Gustavo Gili.

Brecht, B. (1928). La Ópera de los Tres Centavos.

Chaves, N. (2006). El diseño invisible. Siete lecciones sobre la intervención culta en el hábitat urbano. Madrid: Paidós Ibérica.

Chaves, N. (2006). "Qué era, qué es y qué no es el diseño. Intentando dispersar la bruma”, en Actas de Diseño I. Buenos Aires: Universidad de Palermo.

Diseñadores influyentes de la AGI (2001). Ensayos sobre diseño. Buenos Aires: Infinito.

Infobae (18 de mayo de 2016). Villa Zavaleta, la más grande y peligrosa de la ciudad, vista desde un drone. Disponible en: http://www.infobae.com/2016/05/18/1812574-villazavaleta-la-mas-grande-y-peligrosa-la-ciudad-vista-un-drone/ 
Orange, R. (2016, 21 de septiembre). El plan de Suecia contra el consumo desaforado: bajar los impuestos sobre las reparaciones. Eldiario.es. Disponible en: http://www.eldiario.es/ theguardian/desecha-Suecia-reduce-impuestos-reparaciones_0_561344188.html

Pauli, G. (2011). La Economía Azul. Barcelona: Tusquets Editores.

Repair Café (sitio de la iniciativa). Disponible en: repaircafe.org

Toffler, A. (1980). La tercera ola. New York: Bantam Books.

Valdés de León, G. (2010). Tierra de nadie. Una molesta introducción al estudio del Diseño. Buenos Aires: Universidad de Palermo.

Abstract: The function of design should not be questioned or become the debate's focus, since the manifest mission of design is social. It arises from society and its production is oriented and directed towards society. It configured according to the temporary needs of the context from which it emerges. However, addressing the issue of functionality implies defining it more precisely: is it social or humanitarian?

The present work gives a look on these questions, and tries to reflect on the possible "must be" of the design in front of the different professional positions.

Key words: social design - humanitarian design - ephemeral design.

Resumo: A função de design não deveria nem ser qusionada, tampouco converter-se no eixo cetral, já que, a priori sua missão declarada é social. Surge da sociedade e a sua produção está orientada e dirigida para a esta, e é configurado de acordo com as necessidades temporais de contexto dentro do qual emerge. No entanto, abordar a questão da funcionalidade deveria defini-la de maneira mais orecisa: é social ou humanitário?

Este artigo fornece uma perspectiva sobre estas questões, e tentar refletir sobre o possível devir do design frente diferentes posicionamentos profissionais.

Palavras chave: design social - design humanitário - design efêmero. 